\title{
A Multi-Agent System for Image Segmentation A Bio-Inspired Approach
}

\author{
Safia Djemame ${ }^{1}$, Mabrouk Nekkache ${ }^{1}$, Mohammed Batouche ${ }^{2}$ \\ ${ }^{1}$ Département Informatique, Université Ferhat Abbes, Sétif 19000, Algérie \\ s_djemame@yahoo.fr \\ nekkache@gmail.com \\ ${ }^{2}$ Computer Science Department, \\ CCIS-King Saud University, Riyadh, Saudi Arabia \\ batouche@ccis.edu.sa
}

\begin{abstract}
We explore the artificial life domain which opens new horizons to find bio-inspired solutions to image processing problems. Besides the ants which were successfully used, we investigate a new approach based on the interaction of social spiders, where we adapt the collective web weaving to an image segmentation on regions. Spiders were successfully used to extract regions on grey level images. We resume this method and try to adapt it for an image segmentation. The spiders which are considered as simple, autonomous agents, in limited perception, communicate by stigmergy of the silk that they weave, and so build several webs comparable to regions on an image.
\end{abstract}

Keywords: multi-agent systems, image segmentation, artificial life, emergence, stigmergy

\section{INTRODUCTION}

The image segmentation is the heart of any vision system and is an important stage in the image analysis process. It has for objective to supply a description of objects contained in the image by the extraction of various visual indications such as the objects edges, homogeneous regions, $3 \mathrm{D}$ objects. They will be then run for a symbolic description of the scene allowing interpretation and possibly decision-making. Until this day, there is no universal method for image segmentation. Any technique is effective only for a given image type, for a given application type, and in a given context. Because of these constraints, the diverse segmentation strategies which were proposed asserted their incapacities and their limitations, thus it is necessary to investigate new horizons and to find new more flexible and more effective methods.

This paper is organized as follows: the first section gives an overview about artificial life and multiagent systems, their utilities for image segmentation. The second section presents some related works with this subject. The third section explains the social spiders method. The following section presents the method used for segmenting an image before we provide an experimental assessment of the method. Finally, we will conclude and propose some ideas for further works.

\section{ARTIFICIAL LIFE AND MULTI-AGENT SYSTEMS}

For some years, the computer researchers found in the artificial life a source of inspiration for the conception of new computer systems. It is a question of drawing from the behavior of the natural beings new approaches for difficult problems resolution. The role of the computer specialist is to observe and to understand mechanisms and processes then extract from these studies implantable models on machines. The results can be validated with regard to those observed in the nature.

In Biology for example, a lot of natural systems consisted of autonomous simple individuals show capacities to make tasks qualified complex without global control. Furthermore, they can adapt themselves to their environment either to survive, or to improve the functioning of the collective.

It is the case of social insects colonies [3] such as termites, ants, bees [1], or spiders and mouses [2] which effectively show remarkable capacities to make tasks such as: the construction of complex nests, the construction of bridges, food research, prey capture ...The study of flight collective movements of migratory birds or fish shoals watch also that the collective task is the result of interactions between autonomous individuals [11]. . 
All these natural systems present a common point: the emergence of a collective and complex global behavior from simple interactions between simple individuals endowed with a very reduced intelligence and possessing only a very partial vision of their environment [1]. This emerging behavior allows them to resolve collectively very complex problems. So saw the day much tools such as: artificial neural networks (ANN), the genetic algorithms (GA), the multi-agents systems (MAS), swarm based computing. These new methods have in common the qualities inherited from their natural inspiration source: the robustness, the tolerance at noises, the flexibility and the adaptability.

A MAS is a distributed system consisted of a group of agents who interact in an environment. There are two categories of agents: cognitive and reagent. The cognitive agents have a global sight on their environment and know the purpose for which they cooperate. On the other hand, a reagent agent has only a vision limited by his environment, he reacts to stimuli and can modify his environment by adding or by deleting information. The reagent agents do not know the global function of the system. This task appears according to the agents interaction. Insect colonies like ants or spiders are a good example of reagent agents: each knows locally the work which he has to carry out, but ignores everything of the global complex task for which the collective of agents works.

\section{RELATED WORKS [9]}

Ramos and Almeida [10] have explored the idea of using a digital image as an environment for artificial ant colonies. They observed that artificial ant colonies could react and adapt appropriately their behavior to any type of digital habitat. Ramos et al. [11] also investigated ant colonies based data clustering and developped an ant colony clustering algorithm which he applied to a digital image retrieval problem. By doing so, they were able to perform retrieval and classification successfully on images of marble samples. Liu and Tang [8] have conducted similar works and have presented an algorithm for greyscale image segmentation using behavior-based agents that self reproduce in areas of interest. He and Chen [5] have provided an artificial cell model. In their model, each life is one individual unit, called a cell, which adheres to one pixel in the image. All the cells bear similar structures but mutations may occur during the process of reproduction due to the influence of the environment. Hamarneh et al. [4] have shown how an intelligent corpus callosum agent, which takes the form of a worm, can deal with noise, incomplete edges, enormus anatomical variation, and occlusion in order to segment and label the corpus callosum in 2D mid-sagittal images slices in the brain. Bourjot et al. [2] have explored the idea of using social spiders as a behavior to detect the regions of the image. The principle is to weave a web over the image by fixing silks between pixels. The biological model of spiders was detailed in [4]. This method, by its originality and its novelty represents our center of interest and our work considers as a continuation of [2] and [9].

\section{SOCIAL SPIDERS METHOD [2]}

\subsection{The biological model}

Among the thousands of spider species in the world, only about fifteen species can be qualified as social spiders. Anelosimus eximius is a species of social spider which can be found in French Guiana. The individuals live together, share the same web and cooperate in various activities such as brood care, web weaving, hunting, .... On the web, spiders are gathered in small clusters under the vegetal sheets included in the web and distributed on the whole of the silky structure. Figure 1 shows such a society and its web. Despite their apparent individual simplicity, these spiders are exhibiting interesting collective behavior during prey capture and web weaving.

Anelosimus eximius are small animals $(5 \mathrm{~mm})$, able to collectively build silky structures bigger than ten $\mathrm{m}^{3}$ that always respect architectural properties whatever the biological environment is. Webs are not geometrical but twofold: an horizontal hammock and an aerial network of silk lines. Silk attraction has been observed, a temporal succession of solitary spiders on the same partially achieved web can successfully lead to a functional one. Silk is attractive, therefore spiders have the tendency to follow silk draglines rather than moving to adjacent stakes. 


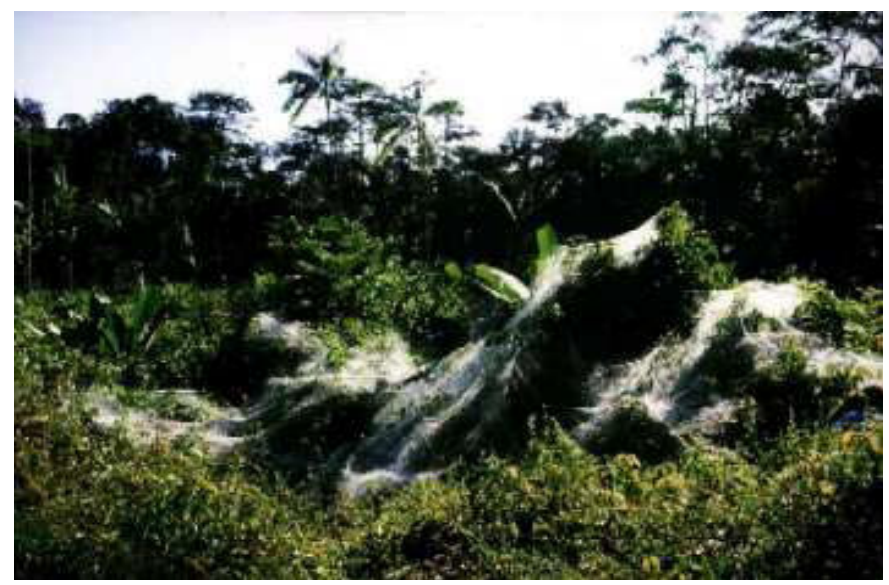

FIGURE 1: A spider colony and its web

Social spiders have been defined by the biologists to present stigmergic process like social insects. The characteristics of these societies and the importance of the silk in the various behavior have created a different model from the social insects one. During their cycle, social spiders have the abilities to fix silks, move forward and move back. This model has characteristics which sufficiently distinguishes the levels of the realized spots, the society organization and the communication supports. Indeed, social spiders correspond to an interest model for three reasons:

1) Social spiders do not present any specialization in morphology and Ethology;

2) An isolated social spider presents behavioral characteristics very close to lonely species;

3) Social spiders show spectacular organization and cooperation forms, in particular, the web construction and the

prey capture or its transportation phenomenon.

\subsection{The Computational Model}

The computational model of simulation is described according to multi-agent concepts: environment, agents and their behavior, and interactions.

\section{Environment:}

It corresponds to a grey level image. It is represented by a two dimensional array whose elements are the pixels of the image. Each pixel is featured by its grey level and by the list of draglines already fixed on it. Initially, the environment contains no draglines, the agents will add draglines during runtime.

\section{Agents}

We can design spiders as agents. Spiders are reactive agents. They are defined by an internal state composed of a set of parameters values, a current position and the last pixel where a spider has silked. These spiders have also an ability to move in the environment, to fix a silk and to come back. Spiders which detect the same region can be grouped in a set called a colony. Spiders of a same colony share the same set of parameters values. Spiders try to move through their 8-neighborhood, they prioritize the nonsilked pixels and try to fix silks on them. If they fail, they move back to the last fixed silk. At the end of the process, groups of spiders are formed and the web they weave is assimilating to a region on an image;

\section{Movement}

In the spider model, it is based on stigmergy, this is a way to achieve coordination without any explicit reference to the task being performed: the past actions put traces in the environment, and these traces favor in return some actions among others. In this case, the modifications are the apparition of silk draglines. It provides new possibilities for movement and they are favored by silk attraction.

\subsection{Experimental setup}

The test presented after was carried out with a real image. The picture resolution is $256^{\star} 256$, it has been obtained from a CCD camera, and has not been preprocessed. 

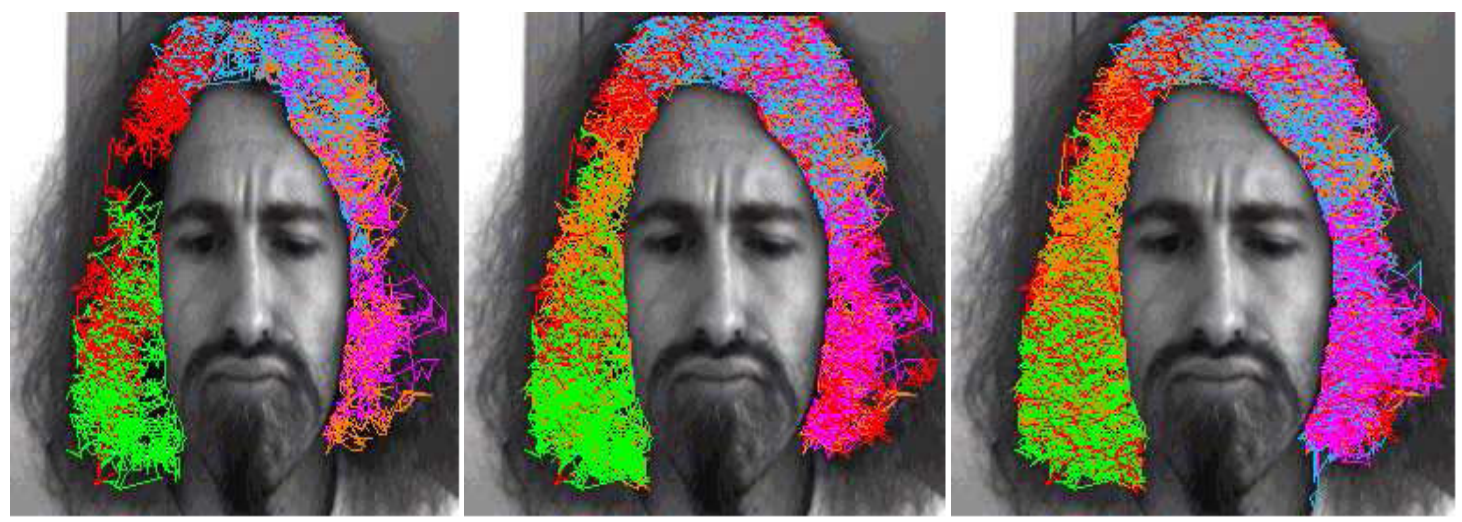

FIGURE 2: Web resulting from the extraction of hair (5 agents after 5000, 10000, 20000 cycles)

A first conclusion is that results confirm the relevancy of this approach since it is able to extract properly various kinds of regions from real images, one of its major advantages is that the same simple behavior is used, and only the individual parameters determine the extracted regions.

\section{SEGMENTATION METHOD [9]}

The multi-agent system is composed of an environment and a set of spider-agents. For segmentation purpose, environment is created from a given grayscale picture: it is a matrix of gray pixels. System and agents have a life cycle. This life cycle is transposed to a step. The number of steps to be executed is given by the user. Each spider computes the three functions. Its complexity is about $\mathrm{O}($ Ite.N), where Ite is the number of iterations and $\mathrm{N}$ the number of spiders.

Algorithm 1 Segmentation method

Require: Pixels: Matrix of pixels 2 N2, Width and Height $2 \mathrm{~N}$, Ite $2 \mathrm{~N}$ and

Conf: Configuration parameters

1: scuts[Width][Height]: Draglines matrix

2: Create colonies and spiders from the Conf

3: while Ite - - >0 do

4: for Each spider $s$ do

5: Movement(s, Pixels, scuts)

6: Silkfixing(s, scuts)

7: Comeback(s)

8: end for

9: end while

\section{Environment}

Environment is a matrix of gray pixels.

Each pixel is a position for spiders and

allows them to access to other pixels.

Neighborhood of a pixel $p$ is defined in

two ways (figure 1):

- The 8 pixels around $p$, which are named Neigh.

- All pixel linked to $p$ by a dragline, which are named SCuts.

Set of all reachable pixels are named Access.

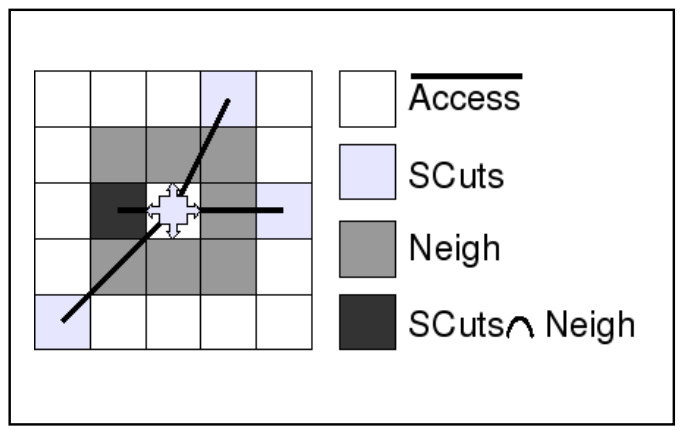

FIGURE 3: Neighborhood of a pixel

\section{Movement}

At first, a spider has to choose to move towards a pixel Scuts or Neigh, this choice is made according to the value of a simulation parameter: pdraglines. Two other parameters: attractself and to attractother measure respectively the probability of movement of an agent towards a dragline weaved by a spider of the same colony, or another colony. 
There, two other parameters are used: reflevel and selectivity. Reflevel corresponds at the grey level of the region to be detected. Selectivity is the probability to fix a dragline with a level pixel of different grey. It is a gaussian distribution of average reflevel.

\section{Comeback}

This behavioral item allows a spider to return on the last weaved dragline. This action depends on the probability value expressed by backprobability parameter. This action aims at detecting the regions of interconnected pixels and at preventing the spiders from getting lost far from the weaved regions.

\subsection{Dynamic system}

It is based on stigmergy: every spider leaves in the environment informations which will be used by the other agents or by herself during a next cycle. The image segmentation appears from the global behavior: after a certain number of cycles, the silk draglines are weaved. The degree of a pixel represents the number of entering draglines or going out of this pixel.

\subsection{Experimentation}

In this paragraph, we present the results obtained on MRI images (magnetic resonance imaging), and the comparisons with two other segmentation methods. The image test was obtained by a simulator of intellectual MRIs called BrainWeb, A slice of this model is presented in figure 2.

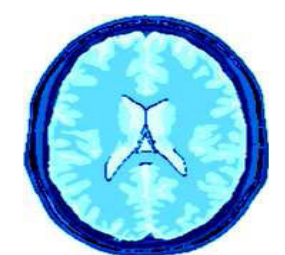

FIGURE 4: slice of the 3D model

The parameters of the model to be used in this study are:

- Modality: T1

- Slice thickness: $1 \mathrm{~mm}$

- Noise: $3 \%$

- Intensity non-uniformity: $20 \%$

- Dimensions: 181 x 217 x 181vx

At first, a segmentation is made on a $2 \mathrm{D}$ image (figure 3). Afterward, the same process will be applied to a $3 \mathrm{D}$ image (figure 2) to test the efficiency on complex images.

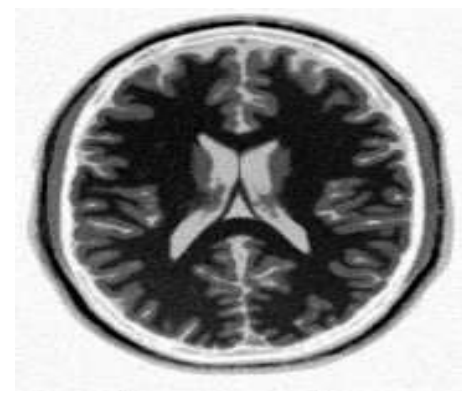

FIGURE 5: 2D slice: $181 \times 217 \mathrm{px}$ 


\subsection{Comparison methods}

In this section, we will compare the social spiders method with other segmentation methods. These comparisons will allow us to determine whether the social spiders method brings something positive compared to traditional segmentation methods.

We will use these comparisons on two other methods:

- A classification method by thresholding: the OTSU method;

- A region-based method: the region growing method.

To compare these methods, we need to establish criteria to be used on all test images. We will compare the results on several points:

1. The number of regions;

2. Correspondence between the regions of the model and the segmentation result;

3. Execution time.

\subsubsection{Region growing}

The Region growing method consist on building a region from one chosen pixel and then adding recursively neighbors whose grayscale difference with the original pixel is below a threshold.

This method tries to grow an initial region by adding pixels that do not belong to any region but to the pixels neighborhood already in the region and whose grayscale is sufficiently close to the area. When it is not possible to add pixels, we create a new region with a pixel that has not been selected yet, then we grow the region.

The method ends when all the pixels were chosen by a region. To automatize the process of this algorithm, the threshold was chosen as the local minima of the image histogram.

\subsubsection{OTSU}

OTSU [9] is a multi-level thresholding method. Its aim is to determine, for a given number of regions, the optimum values of different thresholds based on the variance of subdivisions created.

The basic method consists on separating the foreground from the backgroud. In this case, we search the optimal threshold to split the pixels in two regions. For a threshold $t$, it is possible to compute the betweenclass variance. This measure is derived from the average intensity $\mu 1, \mu 2$ and $\mu$ of classes $[0 ; t],[t+1 ; L]$ and $[0 ; L]$ where $L$ is the maximum intensity. The OTSU method shows that the optimal threshold $t$ is obtained for a betweenclass variance. The method consists on computing the variance for all possible thresholds (t $2\{1 ; \ldots ; L-1\})$ and determining its maximal value.

The execution time to be given comes from the simulation of the methods on a machine equipped with two Intel (R) Xeon (R) E5345 (8 cores having $2.33 \mathrm{GHz}$ ) and 8GB of RAM. The operating system of this machine is a Linux kernel 2.6.21 $\times 8664$.

\subsection{Results}

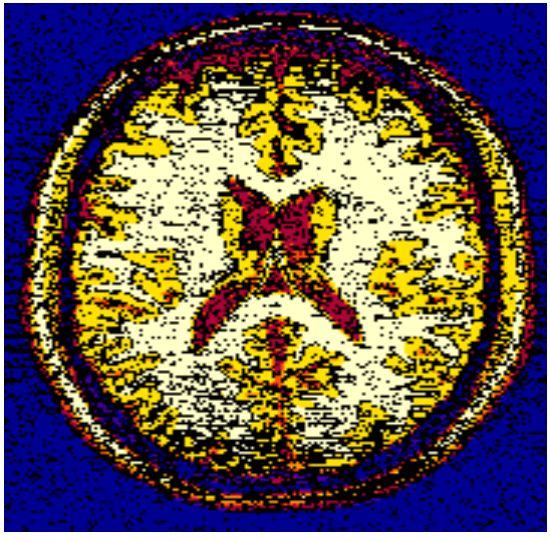

a

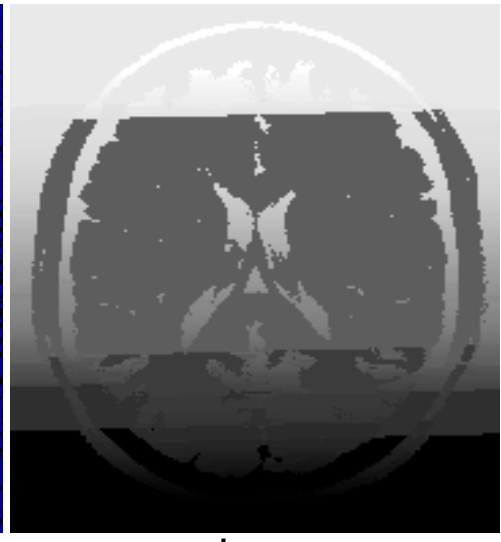

b

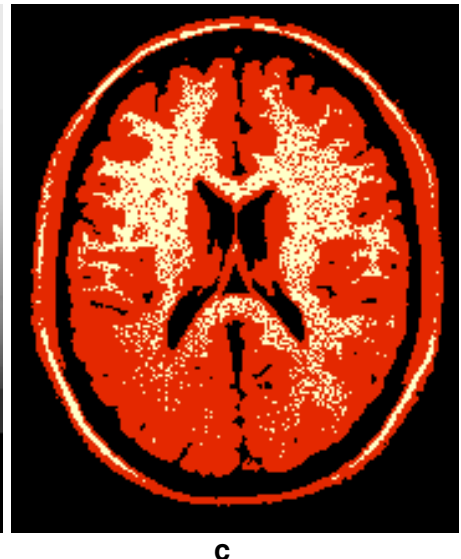

C

FIGURE 6: 2D segmentation of a brain slice: a) Social spiders, b) Region growing, c) OTSU

\begin{tabular}{|c|c|c|c|c|}
\hline & REGIONS & REG > 10 PX & ACCURACY & TIME \\
\hline SOCIAL SPIDERS & 4361 & 434 & $60,8 \%$ & 50 SEC \\
\hline REGION GROWING & 908 & 664 & $46,2 \%$ & 0.5 SEC \\
\hline OTSU & 1875 & 567 & $90,7 \%$ & 0.2 SEC \\
\hline
\end{tabular}

TABLE 1: 2D results 
The Social Spiders method produced the highest number of significant regions, that closest to the actual number of regions. However, it is also the method that produced the greatest number of insignificant regions, meaning that the spiders have oversegmented the image. The execution time of the Social Spiders method is too high compared to the two other methods. Spiders make more time (number of iterations) to find pixels corresponding to their colony. Table 2 shows the results of different segmentations of the image BrainWeb in three dimensions.

\begin{tabular}{|c|c|c|c|c|}
\hline & REGION & REGION > 10 PX & ACCURACY & TIME \\
\hline SOCIAL SPIDERS & 45575 & 13391 & $87,3 \%$ & $8 \mathrm{H}$ \\
\hline REGION GROWING & 7866 & 1634 & $75,4 \%$ & 102 SEC \\
\hline OTSU & 8497 & 865 & $83,9 \%$ & 10 SEC \\
\hline
\end{tabular}

TABLE 2: 3D results

The accuracy displayed by the spiders method is quite correct compared to the other methods. However, a number of regions rather high can be explained by a number of voxels non-detected more important, leading to disconnection of the regions. Unfortunately, this accuracy has a very important consuming time over the execution time. Therefore, as well as for the results on two dimensions images, we get a bad time result. The OTSU method, although with a precision of a few percent lower, produces a good result for a shorter time computation of about 4000 times. In addition, the number of regions produced by the spiders method is greater by a factor of 10 compared to the other methods.

\section{CONCLUSION AND FUTURE PERSPECTIVES}

In this work, we exposed a new segmentation method based on a reagent multi-agents system, composed of a set of spiders which make the extraction of regions on an image by weaving a web. Every group of spiders has a set of parameters; the number of cycles and the number of spiders is fixed by the user. We compared the results obtained with two other segmentation methods: by growth of region and OTSU, we focused on the execution time, the number of obtained regions and the exactness of the methods. We particularly ended that the spiders method allowed to extract a significant number of regions and looked a relatively long time execution. This drawback could be by-passed by using parallel architecture, indeed, the various agents who execute the same algorithm could be distributed on several processors, architecture SIMD (Single Multiple Instruction Dated) with graphic processors (type GPU: Graphical Processing Unit) would be suited and would reduce considerably the execution time.

We have seen that the second drawback of the spiders method is producing an oversegmentation of the image. It is possible to solve this problem by initiating the algorithm by merging insignificant regions to ratach them to significant regions or merging them as appropriate. Merging operation is not an expensive treatment time processor, therefore, adding a post-treatment to the social spiders method adds a negligible computation time compared to the method itself.

Some solutions have been considered to improve the method. They will be the object of future works:

The spiders seem sensitive to the topology of the image, so it is possible to guide spiders movement with a gradient or a laplacian. Indeed, these measures will provide informations on the possible presence of contours. It would be then possible to use spiders in two ways:

1. Gradient would be repellent which would partition a colony of spiders in a region;

2. On the contrary, the gradient could have an attractive effect. In this case, spiders would be used to detect the contours of regions.

The method has been used without other modification than the extension of the neighborhood. Therefore, there is no optimization related to adding an extra dimension. However, the BrainWeb segmentation model showed that the method was capable to produce a result with good accuracy.

Optimizations are needed to reduce the number of regions produced and specially to reduce the time process of the method.

\section{REFERENCES.}

[1] Bonabeau E., Theraulaz G. : Auto-organisation et comportements collectifs : la modélisation des sociétés d'insectes, Auto-organisation et comportement, Editions Hermès, (1997).

[2] Bourjot.C, Chevrier.V, Thomas.V: A new swarm mechanism based on social spiders colonies: from web weaving to region detection. Web intelligence and agent systems: an international journal, vol 1, pp 47-64, WIAS (2003).

[3] Camazine.S, Deneubourg.J.L, Franks.N.R , Sneyd.J, Theraulaz.G, Bonabeau.E : Self-Organization in Biological Systems, Princeton University Press,( 2002). 
[4] Krafft.B: Colony distribution of the social spider Anelosimus eximius (Araneae, Theridiidae) in French Guyana. Insectes Sociaux 36 : 173-182. (1989)

[5] G. Hamarneh, T. Mclnerney, and D. Terzopoulos. Deformable organisms for automatic medical image analysis. Medical Image Analysis, pages 66-76, 2001.

[6] H. He, Y. Chen. Artificial life for image segmentation. Internation Journal of Pattern Recognition and Artificial Intelligence, volume 15, Issue 6:989-1003, 2001.

[7] B. Jahne. Digital Image Processing. Springer, sixth edition, 2005.

[8] Liu.J, Tang.Y, Adaptive image segmentation with distributed behavior based agents, in: IEEE Transactions on Pattern Analysis and Machine Intelligence, Vol. 21, No. 6, pp 544-551, juin (1999)

[9] Moussa R., Beurton-Aimar M., Desbarats P., Savin G., « image segmentation using socials agents », research report hal-00359897, LABRI (Bordeaux), 2009.

[10] V. Ramos, F. Muge, and P. Pina. Self-organized data and image retrieval as a consequence of interdynamic synergistic relationships in artificial ant colonies. Frontiers in Artificial Intelligence and Applications, Soft Computing

Systems - Design, Management and Applications, 2nd International Conference on Hybrid Intelligent Systems, IOS Press, in Javier Ruiz-del-Solar, Ajith Abraham and Mario Kppen (Eds.), volume 87, ISBN 1 5860 32976:500-509,

Santiago, Chile, December 2002.

[11] Theraulaz G., Spitz F. : auto-organisation et comportement, Hermés, Paris, 1997. 